\title{
Nutrition data use and needs: Findings from an online survey of global nutrition stakeholders
}

\author{
Audrey J Buckland', \\ Andrew L Thorne-Lyman ${ }^{1}$, \\ Tricia Aung ${ }^{1}$, Shannon E King ${ }^{1}$, \\ Renee Manorat ${ }^{2}$, Laura Becker ${ }^{2}$, \\ Ellen Piwoz ${ }^{3}$, Rahul Rawat ${ }^{3}$, \\ Rebecca Heidkamp ${ }^{1}$
${ }^{1}$ Department of International Health, Johns Hopkins Bloomberg School of Public Health, Baltimore, Maryland, USA
${ }^{2}$ Results for Development, Washington, D.C., USA
${ }^{3}$ Bill \& Melinda Gates Foundation, Seattle, Washington, USA

\begin{abstract}
Background There is growing global demand for country-specific information to track nutritional status and its determinants, including intervention coverage. Periodic population-based surveys form the backbone of most national nutrition information systems. However, data on the coverage of many nutrition specific and sensitive interventions remain sparse.

Methods An online survey was administered to the international nutrition community in 2018 through relevant listservs and professional networks to characterize their use of nutrition-related indicators and data sources. Respondents were asked about their professional background, access and use of specific indicators and data sources in the previous year, and unmet data needs. Results were tabulated by respondent characteristics and $\chi^{2}$ tests used for statistical testing.
\end{abstract}

Results Complete survey responses were received from 235 respondents, the majority from non-governmental organizations and research communities, and few from governments. Demographic Health Surveys (DHS) were the most frequently accessed country-specific data source and the Global Nutrition Report (GNR) was the most accessed consolidated data source, each accessed by approximately $75 \%$ of respondents. Respondents with a multi-country focus were more likely to have accessed DHS than those with a single-country focus $(85 \%$ vs $60 \%, P<0.001)$. Similarly, respondents with a multi-country focus were more likely to have accessed the GNR compared to those with a single-country focus ( $82 \%$ vs $66 \%, P<0.05)$. The most commonly accessed indicators overall were the prevalence of exclusive breastfeeding (69\%), child minimum dietary diversity (66\%), under-5 stunting (65\%), and under-5 wasting (65\%). Reported data gaps included adult and household diet quality indicators $(n=32)$, nutrition-sensitive intervention coverage $(n=25)$, and infant and young child feeding promotion coverage $(n=11)$. Lack of data availability for the desired geographic level (82\%) or demographic group of interest (82\%) and out-of-date data (77\%) were common data challenges experienced by respondents.

Conclusions The survey results highlight the continued need for high-quality, actionable nutrition data to help facilitate progress towards national and global nutrition targets.

There is growing global demand for country-specific information to track population-level nutritional status and its determinants, including the coverage of key nutrition interventions. The 2014 Global Nutrition Report (GNR) called for a "nutrition data revolution" that includes filling data gaps and making information accessible in order to improve accountability and identi- 
fy key areas for action [1]. The Sustainable Development Goal (SDG) to end malnutrition in all its forms by 2030 [2] reinforces the need for high-quality actionable nutrition data to track actions and progress on meeting this SDG.

Periodic population-based surveys form the backbone of most national nutrition information systems. Commonly available household surveys include Demographic and Health Surveys (DHS), the Multi-Indicator Cluster Surveys (MICS), as well as a variety of national and sub-national surveys, including the Standardised Monitoring and Assessment of Relief and Transitions (SMART), micronutrient and dietary consumption surveys. Many countries and development partners are also investing in strengthening routine facility data systems for nutrition by adding indicators to their District Health Information System 2 (DHIS-2) [3-5] and other sector-specific management information systems. However, data on the coverage of many nutrition specific and sensitive interventions remain sparse [6]. Intervention coverage is the proportion of the population who received a service out of those who were eligible. A review of the DHS and MICS core questionnaires - a set of survey questions implemented in every country - found that only 8 of the $23 \mathrm{WHO}$-recommended nutrition interventions are captured by these tools [7].

Adding new indicators to multi-topic household surveys (eg, DHS, MICS) and administrative systems is a resource-intensive process. It increases the data collection, training, and supervision burden and can compromise data quality due to data collector and/or respondent fatigue. Changes to administrative systems often require printing and distributing new registers to facilities, retraining of staff and changes to data tabulation and analysis. To justify these investments, we need clear evidence that potential users demand the information. To help characterize that demand, we conducted an online survey among nutrition professionals working in low- and middle- income countries (LMIC). The survey had two aims: 1) Identify the nutrition indicators and data sources currently used by stakeholder groups; 2) Identify the unmet nutrition information needs of these data users.

\section{METHODS}

\section{Data collection}

The online survey was created using Qualtrics software (Qualtrics, Provo, UT, USA), Version June 2018. The goal of our sampling was to get as many participants from the broader nutrition community as possible at all levels, and so we disseminated the survey through as many professional networks as possible. These included online nutrition-focused listservs with self-elected members (ie, Ag2Nut, Ag2Nut Ethiopia, CORE Group Nutrition, CORE Group General), institutional listservs (ie, WHO Nutritionlist and SUN), professional networks (ie, SUN, UNICEF, Bill \& Melinda Gates Foundation, Johns Hopkins University), and Twitter. In this convenience sample, potential respondents were first contacted through an introductory email from the Data for Decisions to Expand Nutrition Transformation (DataDENT) study team, then were directed to a web-based portal in Qualtrics. Data were collected from July 16 to August 16, 2018.

Respondents were asked about their professional background, how they use data in their work, which nutrition indicators and data sources they accessed or used in the previous year as well as unmet data needs. Respondents were asked to identify which country-specific and consolidated data sources they accessed in the last 12 months. Consolidated data sources are those that present secondary data from multiple countries, typically using multiple data sources (eg, GNR, UNICEF State of the World's Children Report, Scaling up Nutrition Monitoring, Evaluation, Accountability, and Learning (SUN MEAL)). Several questions allowed for multiple responses. Additional follow-up questions were asked about the data sources used by respondents that reported use of coverage data related to common interventions implemented in many countries, including routine growth monitoring for children, screening for acute malnutrition, treatment for severe acute malnutrition (SAM) or moderate acute malnutrition (MAM), vitamin A supplementation, iron folic acid (IFA) supplementation for women or adolescent girls, multiple micronutrient supplementation for women or adolescent girls, breastfeeding counseling, and complementary feeding counseling. The survey tool is available in Appendix S1 of the Online Supplementary Document.

Results were tabulated and compared across categories of geographic focus (single vs multi-country) and organization using Pearson $\chi$-2 statistics calculated in Stata Version 14.0 (Stata Corporation, College Station, TX, USA). Respondents were not required to respond to all questions and therefore the sample size varies by question. The survey received a "Not Human Subjects Research" determination by the Johns Hopkins Bloomberg School of Public Health Institutional Review Board. 
Table 1. Respondent background characteristics ( $\mathrm{n}=235$ )

\begin{tabular}{|c|c|}
\hline Characteristic & $n(\%)$ \\
\hline \multicolumn{2}{|l|}{ Organization: } \\
\hline Non-governmental organization (NGO) & $70(30 \%)$ \\
\hline United Nations or other multinational agency & $57(24 \%)$ \\
\hline University/research institute & $54(23 \%)$ \\
\hline Government & $27(11 \%)$ \\
\hline Donor & $13(6 \%)$ \\
\hline Private sector & $12(5 \%)$ \\
\hline Other & $2(1 \%)$ \\
\hline \multicolumn{2}{|l|}{ Educational achievement: } \\
\hline Secondary school & $1(0.4 \%)$ \\
\hline Undergraduate & $19(8 \%)$ \\
\hline Masters & $130(55 \%)$ \\
\hline Doctoral & $83(35 \%)$ \\
\hline Other & $2(1 \%)$ \\
\hline \multicolumn{2}{|l|}{ Years of experience: } \\
\hline $0-1$ & $7(3 \%)$ \\
\hline $2-4$ & $38(16 \%)$ \\
\hline $5-9$ & $65(28 \%)$ \\
\hline $10+$ years & $125(53 \%)$ \\
\hline \multicolumn{2}{|c|}{ Decisions made related to nutrition (multiple responses allowed) } \\
\hline Monitoring \& evaluation & $154(66 \%)$ \\
\hline Advocacy priorities & $94(40 \%)$ \\
\hline Strategic program and policy planning & $90(38 \%)$ \\
\hline Implementation & $81(34 \%)$ \\
\hline Program-specific financial management & $51(22 \%)$ \\
\hline Program administration & $48(20 \%)$ \\
\hline High-level financing & $30(13 \%)$ \\
\hline Other & $27(11 \%)$ \\
\hline Technical support & $8(3 \%)$ \\
\hline Research & $5(2 \%)$ \\
\hline
\end{tabular}

\section{RESULTS}

\section{Respondent characteristics}

A total of 264 survey responses were received; 235 with responses providing information beyond the identifiers section. The majority of respondents were from NGO and research communities, with relatively few respondents working in government (Table 1). The sample was highly educated and experienced, with over $90 \%$ of respondents having a master's or doctoral degree. Two-thirds of respondents made nutrition-related monitoring and evaluation decisions in their professional roles. Of the $84 \%$ of respondents who self-identified as a technical expert on nutrition related issues, the most common domains of expertise included infant and young child feeding (IYCF) (74\%), child nutrition (71\%), and maternal nutrition (59\%).

\section{Geographic work focus of respondents}

The survey sample was evenly split between those who worked in a single country (49\%) vs across multiple countries (51\%). Among those who worked in a single country, half said their primary work focus was at the national level and half at the subnational level. Among those who worked across multiple countries, $40 \%$ worked at a global level, 25\% at a regional level (eg, North Africa, Southeast Asia), $22 \%$ at a national level, and $14 \%$ at a subnational level (eg, state, district). Respondents were asked to list all the countries to which their work related in the last year, totaling 116 unique countries. By WHO region, the majority of respondents worked in at least one African country (63\%), followed by South-East Asia (40\%), the Americas (19\%), the Eastern Mediterranean (17\%), the Western Pacific (16\%), and Europe (6\%). The most frequently cited countries were India $(n=57)$, Ethiopia $(n=56)$, Kenya $(n=44)$, Bangladesh $(n=44)$, and Burkina Faso $(n=37)$.

\section{Data for decision-making}

When asked about their current role working with data, $72 \%$ reported that they make decisions using data analyzed by others, $63 \%$ consolidate or analyze data for decision making within their institution, and $49 \%$ consolidate or analyze data for external decision making. Decisions made using these data varied by single and multiple country focus. Respondents working in a single country were more likely to make decisions related to program administration compared to those who worked in multiple countries ( $25 \%$ vs $15 \%, P=0.0499$ ). Those with a multi-country focus were more likely than single-country focus respondents to make strategic program and policy planning decisions ( $49 \%$ vs $26 \%, P<0.001$ ) or high-level financing decisions (18\% vs 7\%, P=0.014).

\section{Access to country-specific and consolidated sources of data}

Most respondents (81\%) accessed at least one country-specific data source (eg, national household surveys, routine facility data systems, surveillance systems) in the last 12 months. Of those who accessed country-specific data in the last year, the DHS [8] were by far the most common (74\%), followed by the MICS (42\%) (Table 2). Respondents with a multi-country focus were more likely to access the DHS in the last year (85\%) compared to those with a single-country focus $(60 \%)(P<0.001)$, a pattern that was even more apparent for the MICS (65\% vs 16\%, P<0.001). Routine facility data sources such as the DHIS-2 and Health Management Information System (HMIS) were less accessed compared to household surveys, accessed only by about 30\% of respondents, with no meaningful difference by respondents working at single vs multiple country levels. However, the DHIS-2 data was accessed more by single-country respondents 
Table 2. Country-specific data sources accessed in the last year by geographic level of focus, multiple responses allowed ( $\mathrm{n}=190$ )

\begin{tabular}{|c|c|c|c|}
\hline \multirow{3}{*}{ COUNTRY-SPECIFIC DATA SOURCES } & \multicolumn{3}{|c|}{ Geographic Scope } \\
\hline & $\begin{array}{l}\text { Single country } \\
\qquad(\mathrm{N}=88)\end{array}$ & $\begin{array}{l}\text { Multi country } \\
\qquad(\mathrm{N}=102)\end{array}$ & $\begin{array}{c}\text { Total } \\
(\mathrm{N}=190)\end{array}$ \\
\hline & No. (\%) & No. (\%) & No. (\%) \\
\hline Demographic Health Survey (DHS) & $53(60 \%)$ & $87(85 \%)^{*}$ & $140(74 \%)$ \\
\hline Multiple Indicator Cluster Survey (MICS) & $14(16 \%)$ & $66(65 \%)^{*}$ & $80(42 \%)$ \\
\hline Other National Nutrition Survey (eg, micronutrient survey) & $39(44 \%)$ & $39(38 \%)$ & $78(41 \%)$ \\
\hline National survey using SMART methodology & $26(30 \%)$ & $49(48 \%)^{*}$ & $75(39 \%)$ \\
\hline National Dietary Intake / Food Consumption Survey & $33(38 \%)$ & $31(30 \%)$ & $64(34 \%)$ \\
\hline Sub-national survey using SMART methodology & $23(26 \%)$ & $39(38 \%)$ & $62(33 \%)$ \\
\hline DHIS-2 / similar online HMIS portal & $29(33 \%)$ & $32(32 \%)$ & $61(32 \%)$ \\
\hline Health Management Information System (HMIS) & $23(26 \%)$ & $30(29 \%)$ & $53(28 \%)$ \\
\hline Household, Income, Consumption \& Expenditure survey & $17(19 \%)$ & $18(18 \%)$ & $35(18 \%)$ \\
\hline National food security "hot spot" monitoring system/FEWS-NET & $14(16 \%)$ & $20(20 \%)$ & $34(18 \%)$ \\
\hline World Bank Living Standard Measurement Studies (LSMS) & $4(5 \%)$ & $25(25 \%)$ & $29(15 \%)$ \\
\hline WFP Food Security Monitoring System (FSMS) & $6(7 \%)$ & $20(20 \%)$ & $26(14 \%)$ \\
\hline Other survey specific to program or policy & $11(13 \%)$ & $13(13 \%)$ & $24(13 \%)$ \\
\hline WFP Comprehensive Food Security and Vulnerability Assessments (CFSVA) & $6(7 \%)$ & $17(17 \%)$ & $23(12 \%)$ \\
\hline Other national household surveys with nutrition data & $11(13 \%)$ & $10(10 \%)$ & $21(11 \%)$ \\
\hline Service Provision Assessment (SPA) & $6(7 \%)$ & $15(15 \%)$ & $21(11 \%)$ \\
\hline WFP Emergency Food Security Assessment (EFSA) & $6(7 \%)$ & $13(13 \%)$ & $19(10 \%)$ \\
\hline Demographic surveillance sites (DSS) & $12(14 \%)$ & $7(7 \%)$ & $19(10 \%)$ \\
\hline Other facility survey & $9(10 \%)$ & $7(7 \%)$ & $16(8 \%)$ \\
\hline Other national surveillance system & $4(5 \%)$ & $6(6 \%)$ & $10(5 \%)$ \\
\hline Education MIS & $6(7 \%)$ & $4(4 \%)$ & $10(5 \%)$ \\
\hline WASH MIS & $6(7 \%)$ & $2(2 \%)$ & $8(4 \%)$ \\
\hline Agriculture MIS & $2(2 \%)$ & $1(1 \%)$ & $3(2 \%)$ \\
\hline Other sector data systems & $1(1 \%)$ & $3(3 \%)$ & $4(2 \%)$ \\
\hline Other national data sources & $0(0 \%)$ & $2(2 \%)$ & $2(1 \%)$ \\
\hline
\end{tabular}

${ }^{*} \chi-2, P<0.05$, calculated for data sources used by at least $15 \%$ of respondents.

working at a national level than those working at a subnational level ( $40 \%$ vs $21 \%, P=0.048$ ). The Service Provision Assessment (SPA), a health facility survey, was only accessed by one in ten respondents (11\%).

Most respondents accessed at least one source of consolidated data in the last 12 months (75\%), of which the most common was the GNR [9] (Table 3). The GNR was more likely to be accessed by data users with a multi-country focus ( $82 \%)$ than those with a single-country focus $(66 \%)(P=0.014)$. Multi-country focused respondents were also more likely to access the UNICEF State of the World's Children Report, other UNICEF nutrition data sets, and Countdown to 2030 data products (all $P<0.05$ ).

\section{Access to specific types of indicators}

When asked about types of data on nutritional status accessed in the previous 12 months, the most frequent responses were child anthropometric indicators, with the majority accessing data on child stunting and wasting (Figure 1). In comparison, fewer respondents accessed data on the nutritional status of other populations (adolescents, adults and school children). Data on IYCF practices, including breastfeeding and dietary diversity, were also accessed by most respondents (Figure 2). In contrast, less than a third of respondents accessed data related to the diets of women and adolescents (Figure 3)

For intervention coverage and utilization indicators, more than half of respondents reported accessing data on coverage of breastfeeding counseling and complementary feeding counseling (Figure 4). Household surveys (69\%) and administrative data (39\%) were the most common sources reported by those who accessed breastfeeding counseling indicators (Table 4). The same two sources were reported at similar levels for complementary feeding counseling data. About half of those accessing breastfeeding or complementary feeding counseling coverage data wanted these data collected annually.

Half of the respondents reported accessing data on IFA supplementation during pregnancy (Figure 4). The specific IFA indicators used or accessed varied: any IFA consumed (77\%), minimum number of tablets consumed (58\%), IFA purchased or received (49\%). 
Table 3. Consolidated data sources accessed in the last year by geographic level of focus, multiple responses allowed (n=176)

\begin{tabular}{|c|c|c|c|}
\hline \multirow{3}{*}{ CONSOLIDATED DATA SOURCES } & \multicolumn{3}{|c|}{ Geographic scope } \\
\hline & $\begin{array}{l}\text { Single country } \\
\qquad(N=76)\end{array}$ & $\begin{array}{l}\text { Multi country } \\
\qquad(\mathrm{N}=100)\end{array}$ & $\begin{array}{l}\text { Total } \\
(\mathrm{N}=176)\end{array}$ \\
\hline & No. $(\%)$ & No. $(\%)$ & No. $(\%)$ \\
\hline Global Nutrition Report & $50(66 \%)$ & $82(82 \%) *$ & $132(75 \%)$ \\
\hline UNICEF State of the World's Children Report & $32(42 \%)$ & $68(68 \%) *$ & $100(57 \%)$ \\
\hline UNICEF, WHO and the World Bank Joint Malnutrition Estimates & $22(29 \%)$ & $47(47 \%)^{*}$ & $69(39 \%)$ \\
\hline Other UNICEF Nutrition data sets & $21(28 \%)$ & $46(46 \%) *$ & $67(38 \%)$ \\
\hline FAO The State of Food security and Nutrition in the World & $23(30 \%)$ & $40(40 \%)$ & $63(36 \%)$ \\
\hline World Bank Nutrition Country Profiles & $23(30 \%)$ & $39(39 \%)$ & $62(35 \%)$ \\
\hline $\begin{array}{l}\text { Scaling up Nutrition Monitoring, Evaluation, Accountability and } \\
\text { Learning (SUN MEAL) }\end{array}$ & $25(33 \%)$ & $32(32 \%)$ & $57(32 \%)$ \\
\hline WHO Global Targets Tracking Tool & $18(24 \%)$ & $33(33 \%)$ & $51(29 \%)$ \\
\hline Countdown to 2030 & $16(21 \%)$ & $35(35 \%)^{*}$ & $51(29 \%)$ \\
\hline WHO Global Health Observatory & $16(21 \%)$ & $27(27 \%)$ & $43(24 \%)$ \\
\hline FAO Country Indicators & $11(14 \%)$ & $24(24 \%)$ & $35(20 \%)$ \\
\hline WHO Vitamin \& Mineral Nutrition Information Systems & $10(13 \%)$ & $22(22 \%)$ & $32(18 \%)$ \\
\hline $\begin{array}{l}\text { WHO/UNICEF Joint Monitoring Programme for Water Supply, } \\
\text { Sanitation and Hygiene }\end{array}$ & $3(4 \%)$ & $22(22 \%)$ & $25(14 \%)$ \\
\hline IHME Global Burden of Disease & $4(5 \%)$ & $20(20 \%)$ & $24(14 \%)$ \\
\hline Hunger and Nutrition Commitment Index Global: Country profiles & $6(8 \%)$ & $14(14 \%)$ & $20(11 \%)$ \\
\hline FAO/WHO Global Individual Food Consumption Data Tool (GIFT) & $5(7 \%)$ & $14(14 \%)$ & $19(11 \%)$ \\
\hline IHME Child Growth Failure & $1(1 \%)$ & $10(10 \%)$ & $11(6 \%)$ \\
\hline Other global sources & $1(1 \%)$ & $4(4 \%)$ & $5(3 \%)$ \\
\hline
\end{tabular}

$* \chi-2, P<0.05$, calculated for data sources used by at least $15 \%$ of respondents.

Table 4. Data sources accessed and desired availability for coverage indicators

\begin{tabular}{|c|c|c|c|c|c|c|c|c|}
\hline \multirow{7}{*}{$\begin{array}{l}\text { Growth } \\
\text { monitoring } \\
(\mathrm{n}=92)\end{array}$} & \multicolumn{2}{|c|}{ Data SOUReES accessed, No. (\%) } & \multicolumn{2}{|c|}{ Data sources considered "official", №. (\%) } & \multicolumn{2}{|c|}{$\begin{array}{l}\text { Data Are aVAllabie at the } \\
\text { fhequeneY NeEDed?, No. (\%) }\end{array}$} & \multicolumn{2}{|c|}{ 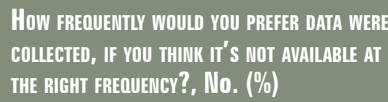 } \\
\hline & Household survey & $63(68 \%)$ & Household survey & $68(74 \%)$ & \multirow{2}{*}{ Yes } & \multirow{2}{*}{$37(40 \%)$} & $2-5 y$ & $9(18 \%)$ \\
\hline & Health facility survey & $36(39 \%)$ & Health facility survey & $27(29 \%)$ & & & Annually & $15(31 \%)$ \\
\hline & Surveillance system & $23(25 \%)$ & Surveillance system & $23(25 \%)$ & \multirow{2}{*}{ No } & \multirow{2}{*}{$49(53 \%)$} & Quarterly & $9(18 \%)$ \\
\hline & Administrative data & $49(53 \%)$ & Administrative data & $44(48 \%)$ & & & Monthly & $8(16 \%)$ \\
\hline & Other & $10(11 \%)$ & Other & $4(4 \%)$ & \multirow{2}{*}{ Missing } & \multirow{2}{*}{$6(7 \%)$} & Other & $5(10 \%)$ \\
\hline & Missing & $6(7 \%)$ & Missing & $6(7 \%)$ & & & Missing & $3(6 \%)$ \\
\hline \multirow{6}{*}{$\begin{array}{l}\text { Acute } \\
\text { malnutrition } \\
\text { screening } \\
(n=105)\end{array}$} & Household survey & $66(63 \%)$ & Household survey & $77(73 \%)$ & \multirow{2}{*}{ Yes } & \multirow{2}{*}{$53(50 \%)$} & $2-5 y$ & $6(12 \%)$ \\
\hline & Health facility survey & $26(25 \%)$ & Health facility survey & $19(18 \%)$ & & & Annually & $10(20 \%)$ \\
\hline & Surveillance system & $34(32 \%)$ & Surveillance system & $26(25 \%)$ & \multirow{2}{*}{ No } & \multirow{2}{*}{$49(47 \%)$} & Quarterly & $15(31 \%)$ \\
\hline & Administrative data & $57(54 \%)$ & Administrative data & $49(47 \%)$ & & & Monthly & $11(22 \%)$ \\
\hline & Other & $15(14 \%)$ & Other & $7(7 \%)$ & \multirow{2}{*}{ Missing } & \multirow{2}{*}{$3(3 \%)$} & Other & $6(12)$ \\
\hline & Missing & $5(5 \%)$ & Missing & $4(4 \%)$ & & & Missing & $1(2 \%)$ \\
\hline \multirow{6}{*}{$\begin{array}{l}\text { Preventive } \\
\text { vitamin A } \\
\text { capsules } \\
(\mathrm{n}=96)\end{array}$} & Household survey & $65(68 \%)$ & Household survey & $66(69 \%)$ & \multirow{2}{*}{ Yes } & \multirow{2}{*}{$64(67 \%)$} & $2-5 y$ & $2(8 \%)$ \\
\hline & Health facility survey & $17(18 \%)$ & Health facility survey & $14(15 \%)$ & & & Annually & $9(36 \%)$ \\
\hline & Surveillance system & $14(15 \%)$ & Surveillance system & $18(19 \%)$ & \multirow{2}{*}{ No } & \multirow{2}{*}{$25(26 \%)$} & Quarterly & $6(24 \%)$ \\
\hline & Administrative data & $55(57 \%)$ & Administrative data & $56(58 \%)$ & & & Monthly & $4(16 \%)$ \\
\hline & Other & $6(6 \%)$ & Other & $7(7 \%)$ & \multirow{2}{*}{ Missing } & \multirow{2}{*}{$7(7 \%)$} & Other & $2(8 \%)$ \\
\hline & Missing & $5(5 \%)$ & Missing & $5(5 \%)$ & & & Missing & $2(8 \%)$ \\
\hline \multirow{6}{*}{$\begin{array}{l}\text { Breastfeeding } \\
\text { counseling } \\
(\mathrm{n}=134)\end{array}$} & Household survey & $93(69 \%)$ & Household survey & $90(67 \%)$ & Yes & $46(34 \%)$ & $2-5 y$ & $11(13 \%)$ \\
\hline & Health facility survey & $29(22 \%)$ & Health facility survey & $22(16 \%)$ & \multirow{4}{*}{ No } & \multirow{4}{*}{$82(61 \%)$} & Annually & $41(50 \%)$ \\
\hline & Surveillance system & $16(12 \%)$ & Surveillance system & $19(14 \%)$ & & & Quarterly & $15(18 \%)$ \\
\hline & Administrative data & $52(39 \%)$ & Administrative data & $56(42 \%)$ & & & Monthly & $12(15 \%)$ \\
\hline & Other & $19(14 \%)$ & Other & $13(10 \%)$ & & & Other & $3(4 \%)$ \\
\hline & Missing & $8(6 \%)$ & Missing & $12(9 \%)$ & Missing & $6(4 \%)$ & Missing & $0(0 \%)$ \\
\hline \multirow{6}{*}{$\begin{array}{l}\text { Complementary } \\
\text { feeding } \\
\text { counseling } \\
(\mathrm{n}=129)\end{array}$} & Household survey & $94(73 \%)$ & Household survey & $93(72 \%)$ & Yes & $44(34 \%)$ & $2-5 y$ & $13(16 \%)$ \\
\hline & Health facility survey & $20(16 \%)$ & Health facility survey & $20(16 \%)$ & & & Annually & $38(48 \%)$ \\
\hline & Surveillance system & $21(16 \%)$ & Surveillance system & $22(17 \%)$ & No & & Quarterly & $14(18 \%)$ \\
\hline & Administrative data & $45(35 \%)$ & Administrative data & $47(36 \%)$ & No & $0(62 \%$ & Monthly & $13(16 \%)$ \\
\hline & Other & $16(12 \%)$ & Other & $13(10 \%)$ & & & Other & $2(3 \%)$ \\
\hline & Missing & $8(6 \%)$ & Missing & $9(7 \%)$ & Missing & $5(4 \%)$ & Missing & $0(0 \%)$ \\
\hline
\end{tabular}




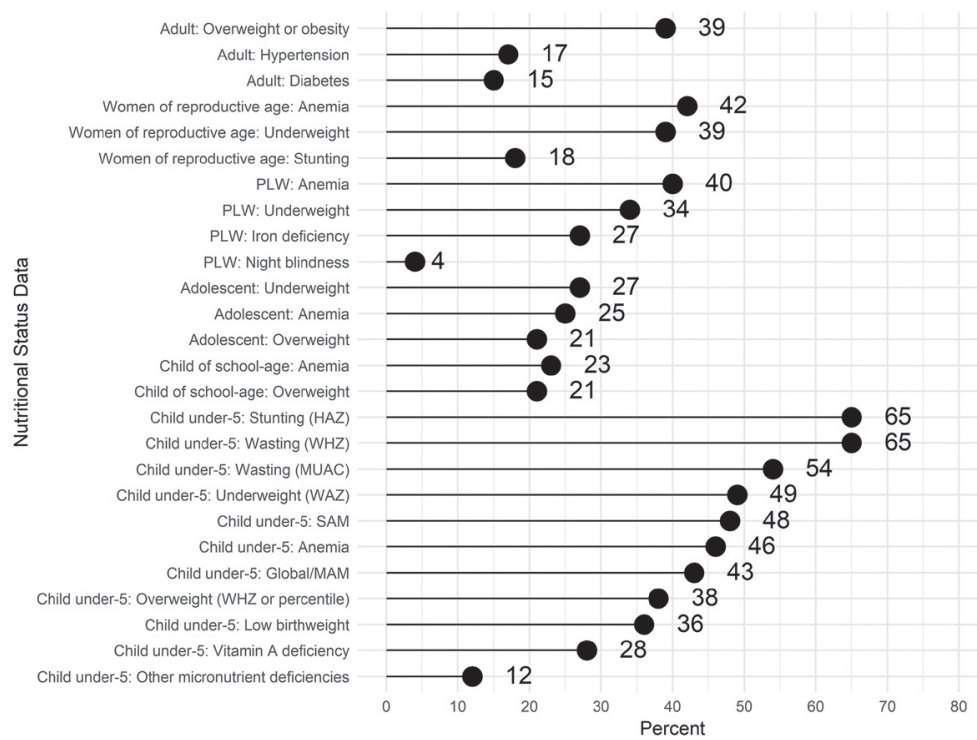

Figure 1. Access or use of nutritional status data $(\mathrm{N}=235)$.

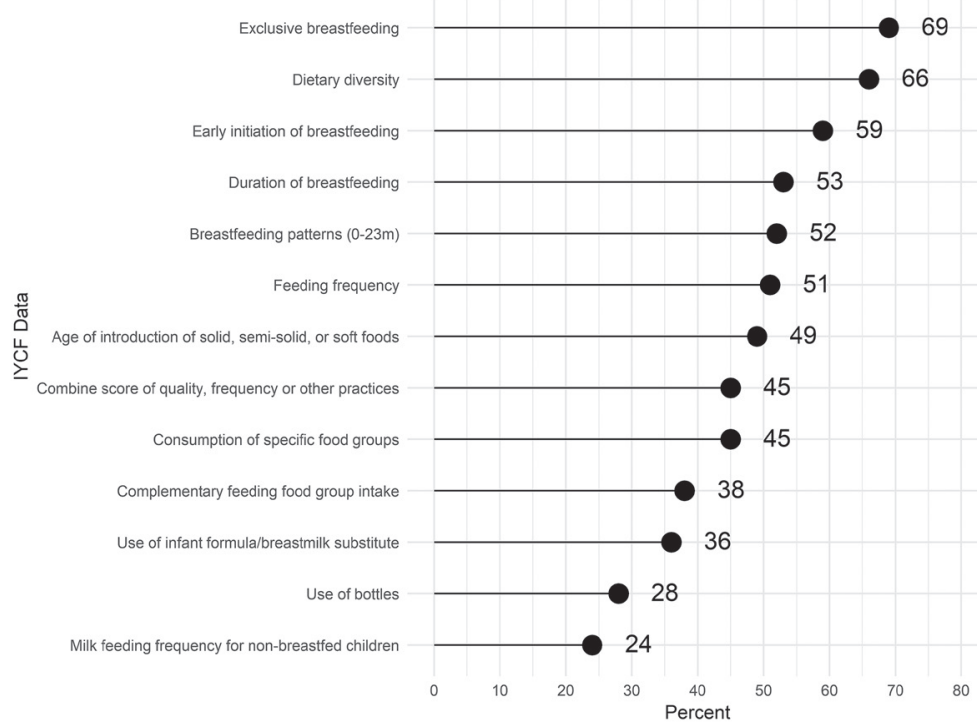

Figure 2. Access or use of infant and young child feeding practices data $(\mathrm{N}=235)$.

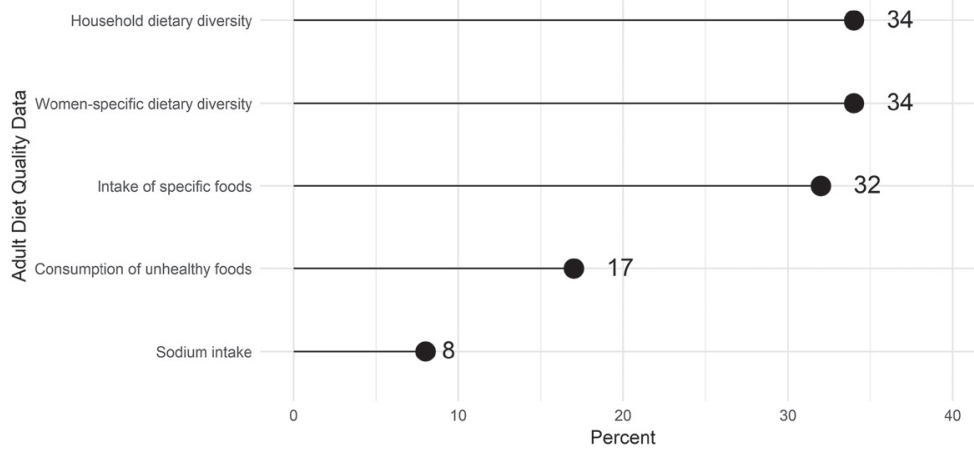

Figure 3. Access or use of adult diet quality data $(\mathrm{N}=235)$. 


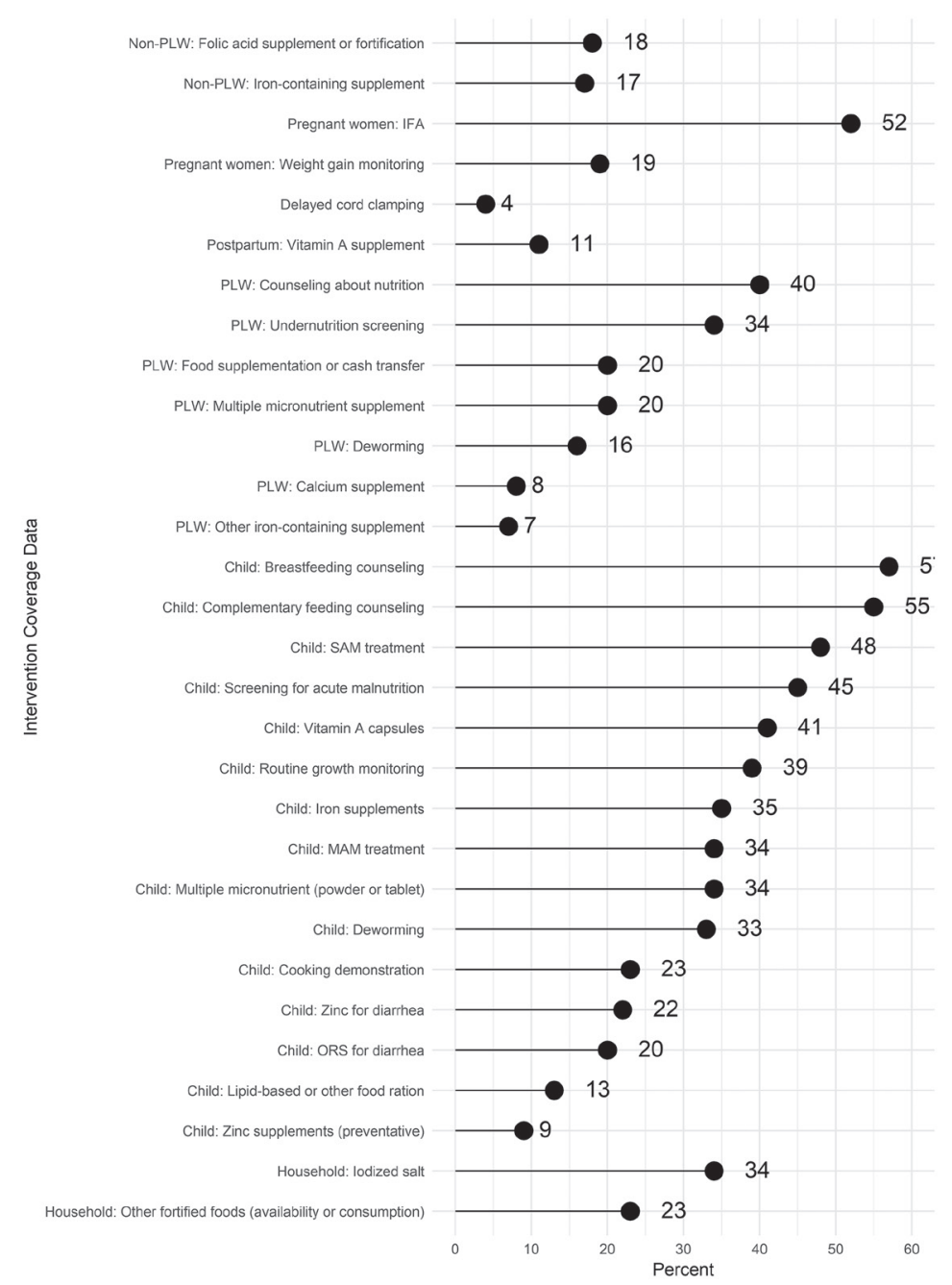

Figure 4. Access or use of coverage or utilization data $(\mathrm{N}=235)$.
About half of respondents reported accessing data on screening for acute malnutrition and/or treatment of SAM. More respondents reported accessing screening data from household surveys than administrative data. In contrast for SAM or MAM treatment, administrative data sources were most accessed (Table 4). For both screening and treatment, about half of respondents who reported accessing the indicators in the last year were satisfied with the current periodicity of available data. The most commonly accessed or used data on nutrition-sensitive interventions were data on water, sanitation and hygiene (Figure 5).

Compared to respondents with a single-country focus, those with a multi-country focus were more likely to access indicators for under-5 wasting, overweight, and vitamin A deficiency, adolescent overweight, women of reproductive age stunting, underweight, and anemia, and gender inequality (all $P<0.05$ ).

\section{Challenges to accessing and using data}

Several challenges to accessing and using nutrition data were reported by respondents. Most respondents reported that data were sometimes or frequently not available at the geographical disaggregation needed (82\%), for the demographic group needed (77\%), or were out-of-date (77\%) (Figure 6). There were no significant differences between multi-country and single-country focus for reported challenges. Responses to an

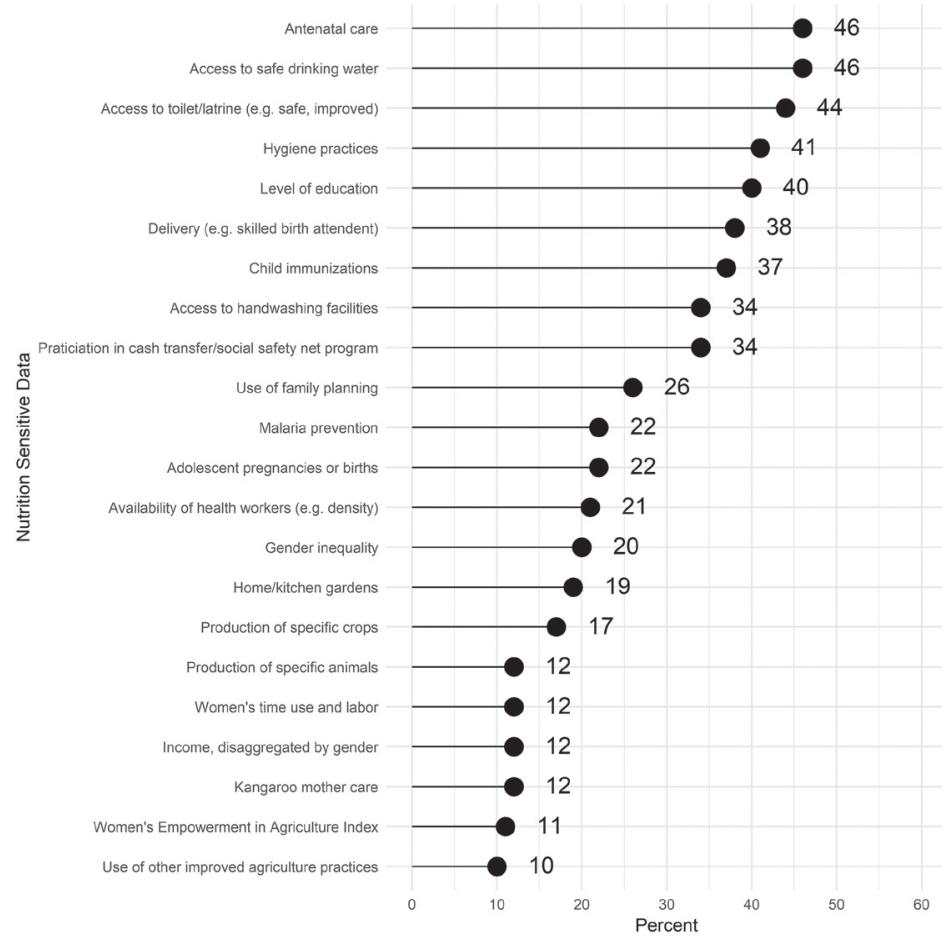

Figure 5. Access or use of nutrition sensitive interventions or drivers data $(\mathrm{N}=235)$. 


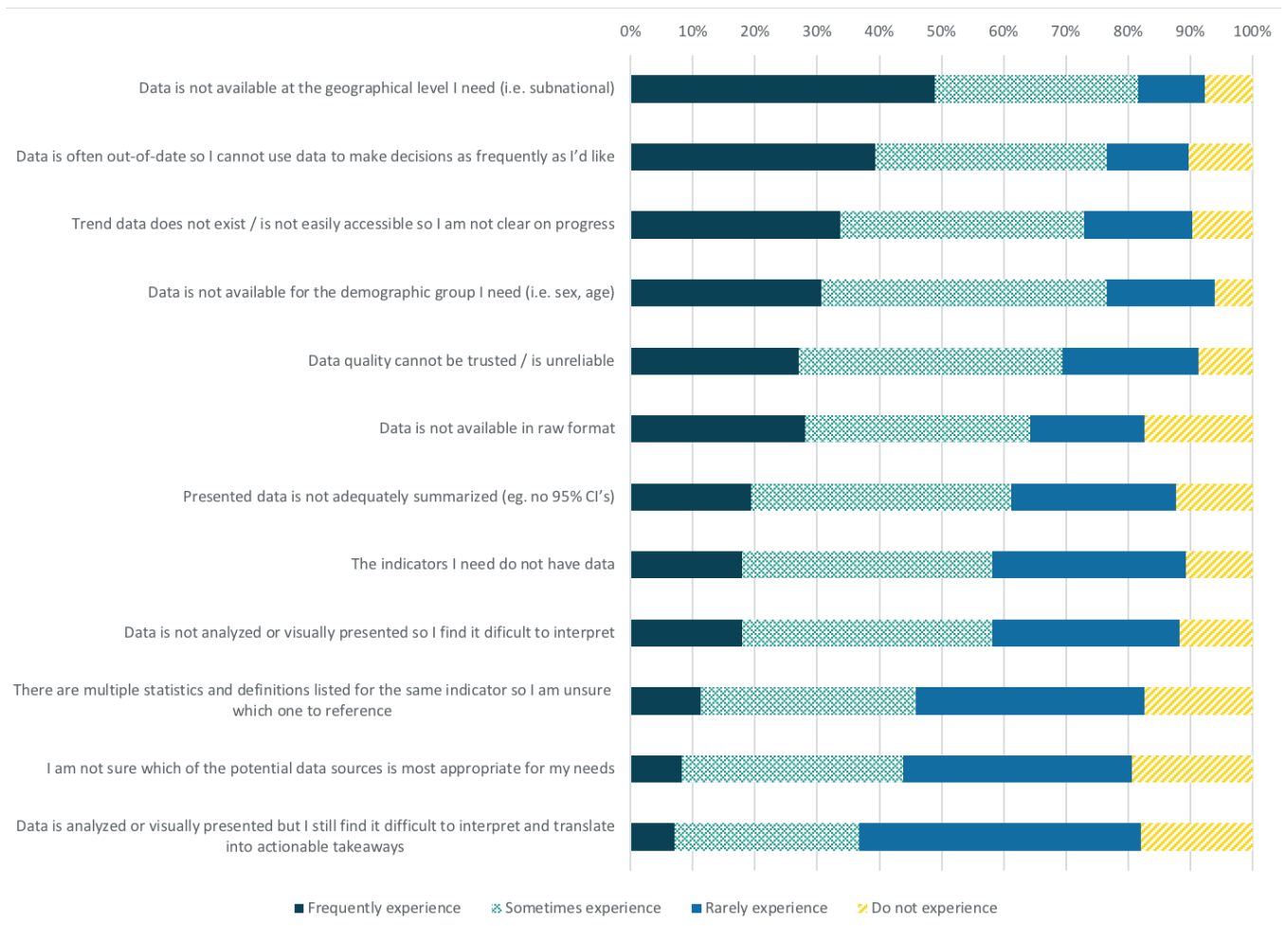

Figure 6. Challenges experienced in accessing and using nutrition data $(n=196)$.

open-ended question about data gaps identified nutrition-sensitive intervention coverage, IYCF promotion and counseling coverage data as priorities.

\section{DISCUSSION}

To our knowledge, this is the first published survey of perceptions of nutrition data use and gaps among stakeholders who work globally in nutrition. Both for stakeholders working in a single country and across multiple countries, the most important data sources were the DHS and the GNR. It is not surprising that the DHS is the most commonly accessed country-specific data source as the DHS program is implemented in more than 90 countries and continues to be a staple for the global health community [10]. We expected respondents with a single-country focus to access the DHIS-2 or HMIS more than those working across multiple countries, but there was no difference. This could be explained by our lack of respondents from LMIC governments. The DHIS-2 has been used in 67 LMICs, and it is possible that we were unable to capture these respondents. Additionally, there might have been more variable access to DHIS2 or HMIS among respondents.

A surprising finding was that respondents reported accessing data on coverage of breastfeeding counseling and complementary feeding counseling at a much higher rate than we expected, particularly given that questions about the coverage of breastfeeding counseling were only recently added to the DHS and MICS and few countries have data on complementary feeding counseling coverage. It is possible that respondents confused indicators of IYCF counseling coverage with indicators of IYCF practices (eg, early initiation of breastfeeding, exclusive breastfeeding, minimum dietary diversity) which have been collected in DHS and MICS for many years and in some administrative systems.

Coverage of antenatal IFA supplementation is a core process indicator of the Global Nutrition Monitoring Framework (GNMF). The results of this survey showed that three different indicators are accessed for antenatal IFA supplementation - IFA consumed, minimum number of tablets consumed, and IFA purchased or received. In 2018, the WHO-UNICEF Technical Expert Advisory group on nutrition Monitoring (TEAM) proposed a simplified definition of antenatal IFA supplementation coverage to accommodate the variability in available data sources: "The percentage of women consuming any iron-containing supplements during the current or past pregnancy within the last 2 years" [11]. 


\section{Limitations}

The generalizability of our survey findings to the broader global nutrition community is uncertain. The survey was disseminated broadly, using established listservs, institutional contacts, and Twitter. This study utilized convenience sampling; given our dissemination channels, those without reliable internet access and those who were not connected to the listservs or professional networks utilized may have been underrepresented. We had very few respondents from LMIC governments, a high priority user group. We also had very few respondents with less than a college education, perhaps suggesting under-representation of front-line workers. Further effort is needed to better understand how national and subnational government stakeholders access and use nutrition data.

\section{CONCLUSIONS AND FUTURE PRIORITIES}

Our findings suggest that nutrition stakeholders have a strong demand for timely data, particularly on nutritional status and IYCF practices and suggest the need to continue and even expand investment in surveys that generate these indicators. Our findings also suggest strong demand for more frequent data collection and more data in general for program coverage indicators including growth monitoring, breastfeeding counseling, and complementary feeding counseling. At the same time, it is important to acknowledge that DHS and MICS multi-topic survey questionnaires are quite lengthy and it is difficult to collect additional indicators without compromising survey quality. This challenge raises questions about what new systems may be needed to support greater frequency of data collection and to potentially support demand for finer granularity in the representativeness of certain indicators at the subnational level.

While there have been ongoing efforts by WHO-UNICEF TEAM to standardize and validate indicator definitions and operational guidance, continued effort is needed, especially to refine data collection tools. These needs are being addressed in part by a number of nutrition-data focused initiatives including WHOUNICEF TEAM, DataDENT, National Information Platforms for Nutrition, SUN MEAL, and Nutrition for Growth [7]. Finally, the data challenges point to a need to improve data literacy so users are better able to decide which data are most appropriate for their needs.

Acknowledgements: The authors would like to thank the participants of the online survey for sharing their experiences with us.

Funding: Funding for this study was provided by the Bill \& Melinda Gates Foundation.

Authorship contributions: AB, ATL, TA, SK, RM, LB, EP, RR, and RH designed this study and survey. SK formatted the survey in Qualtrics, led data management, and contributed to analysis. AB led data analysis and drafted the manuscript with oversight from ATL and RH, and input from all authors. All authors gave final approval of the manuscript to be published.

Competing interests: The authors completed the ICMJE Unified Competing Interest form (available upon request from the corresponding author), and declare no conflicts of interest.

Additional material

Online Supplementary Document

2 United Nations. Trans
Programme, 2015.

3 Kauser A. Improved Data Quality and Use: Dual Goals and HMIS Strengthening. 2016.

4 MEASURE Evaluation. Heath Management Information Systems. 2010. Available: https://www.measureevaluation.org/ resources/training/capacity-building-resources/health-management-information-systems-hmis-1. Accessed: 24 April 2019.

5 Strachan M, Drake M, Rawlins B, Dwivedi V, Levine B, Ly M, et al. Strengthening Health Management Information Systems for Maternal and Child Health: Documenting MCHIPs Contributions Baltimore, MD: Jhpiego Corporation, 2013.

6 Development Initiatives. 2018 Global Nutrition Report: Shining a light to spur action on nutrition. Briston, UK: Development Initiatives Poverty Research Ltd, 2018.

7 Gillespie S, Menon P, Heidkamp R, Piwoz E, Rawat R, Munos M, et al. Measuring the coverage of nutrition interventions along the continuum of care: time to act at scale. BMJ Glob Health. 2019;4:e001290. Medline:31297250 doi:10.1136/ bmjgh-2018-001290 
8 United States Agency for International Development. The Demographic and Health Surveys Program. 2019. Available: https://dhsprogram.com/. Accessed: 29 April 2019.

9 Global Nutrtion Report. About the Global Nutrition Report. 2019. Available: https://globalnutritionreport.org/. Accessed: 30 March 2019.

10 The Demographic and Health Surveys Program. Who We Are. 2019. Available: https://dhsprogram.com/Who-We-Are/ About-Us.cfm. Accessed: 24 August 2019.

11 World Health Organization, United Nations Children's Fund. Developing and validating an iron and folic acid supplementation indicator for tracking progres towards global nutrition monitoring framework targets. Geneva: World Health Organization, 2018. 